\title{
Editorial: Sustainable Hydrogen for Energy, Fuel and Commodity Applications
}

\author{
Xiao-Yu Wu ${ }^{1 *}, Y u$ Luo $^{2 *}$, Franziska Hess ${ }^{3 *}$ and Wojciech Lipiński ${ }^{4 *}$ \\ ${ }^{1}$ Department of Mechanical and Mechatronics Engineering, University of Waterloo, Waterloo, ON, Canada, ${ }^{2}$ National Engineering \\ Research Center of Chemical Fertilizer Catalyst (NERC-CFC), School of Chemical Engineering, Fuzhou University, Fuzhou, China, \\ ${ }^{3}$ Institute of Chemistry, Technical University of Berlin, Berlin, Germany, ${ }^{4}$ Research School of Electrical, Energy and Materials \\ Engineering, The Australian National University, Canberra, ACT, Australia
}

Keywords: hydrogen, low-carbon, sustainability, ammonia, production

Editorial on the Research Topic

Sustainable Hydrogen for Energy, Fuel and Commodity Applications

Hydrogen has an essential role as an energy vector in a sustainable low-carbon future, as shown in Figure 1. It has a high gravimetric density and is an effective energy storage medium for the energy and transportation sectors. Efficient and clean power and heat generation from hydrogen fuel cells and turbines provide new routes to decarbonize the energy and building sectors. Hydrogen is also an important chemical feedstock for various industries such as ammonia and steel to decrease their carbon footprints. Recently, several countries and regions released their hydrogen strategies and roadmaps, such as Canada (Natural Resources Canada, 2020), European Union (European Commission, 2020), and Australia (Council of Australian Governments Energy Council, 2019). It is urgent for the scientific community to provide valuable insights for the transition to the sustainable hydrogen production and utilization by inventing new low-carbon hydrogen production and distribution technologies, quantifying the benefits of hydrogen, and optimizing the hydrogen utilization in various sectors.

This research topic addresses different scientific, technical and economic aspects of using hydrogen for energy, fuel, and commodity applications. The scope of the articles published ranges from hydrogen and hydrogen carrier production to hydrogen utilization in transportation and power sectors. This research topic showcases diverse techniques and capabilities available in the scientific communities to solve hydrogen related problems: literature review and expert opinions, experimental investigation, system-scale modeling, and sector-scale analysis. Authors from China, the United Kingdom, the United States, France, Thailand and Germany contributed to the publications in this research topic.

The benefits of hydrogen depend on how it is produced. Among the 69 Mt hydrogen produced globally (excluding the by-product hydrogen), nearly $99 \%$ is from fossil fuels (i.e., $76 \%$ from natural gas and 23\% from coal) (International Energy Agency, 2019), resulting in significant carbon emissions. It is urgent to develop more sustainable hydrogen production technologies to decrease the associated carbon intensity, as the global demand for hydrogen rises. In this research topic, Zhang et al. studied the system optimization of a biomass-based hydrogen and electricity co-production system. Feedstocks such as wood chips, daily manure, sorghum, and grapevine pruning waste were analyzed. In their design, the hydrogen production system is integrated with an organic Rankine cycle to utilize the high temperature waste heat from the biomass gasifier for electricity generation. An optimal solution predicts a hydrogen yield of $39.31 \mathrm{~mol} / \mathrm{kg}$ and electricity generation of $0.99 \mathrm{kWh} / \mathrm{kg}$ using wood chips as the biomass feedstock, with the hydrogen yield and electricity generation equally important in the optimization. Chuayboon et al. carried out an experimental study on syngas and hydrogen coproduction from methane partial oxidation and water splitting, respectively in a solar-driven

thermochemical redox cycle. The ceria-based reticulated porous ceramics were used as the oxygen

for Energy, Fuel and

Commodity Applications.

Front. Energy Res. 9:698669.

doi: 10.3389/fenrg.2021.698669 


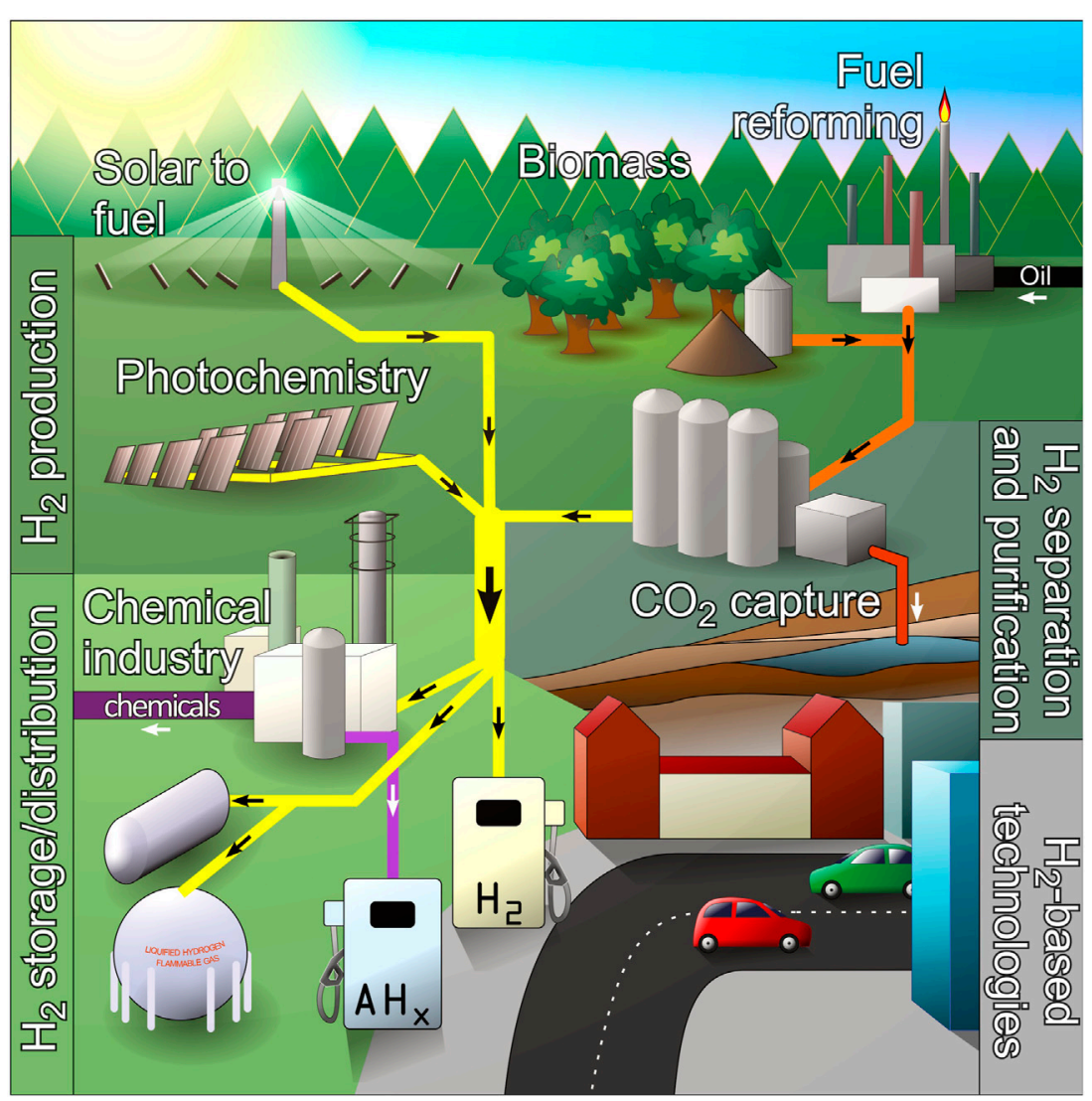

FIGURE 1 | Schematic of an integrated low-carbon economy using various hydrogen technologies, including production, storage/distribution, separation/ purification, and utilization. The flows of energy or species are shown in color: fossil fuels (black), electricity (yellow), reforming and gasification products (orange), carbon dioxide (red), and chemicals and hydrogen carriers (purple).

carrier in a $1.5 \mathrm{~kW}_{\text {th }}$ prototype solar reactor. Solar-to-fuel efficiency was in the range of $2.9-5.6 \%$. While methane was used in this proof-of-concept study, other renewable gaseous feedstock such as biogas can be used to replace methane to produce a purely renewable and carbon neutral solar-fuel.

Various molecules such as methanol and ammonia can serve as hydrogen carriers to facilitate the hydrogen distribution, especially over long distances. In this research topic, Ghavam et al. carried out an extensive literature review on sustainable ammonia production. Both the technologies for hydrogen feedstock production and ammonia synthesis were summarized and compared based on criteria such as environmental impact, efficiency, cost effectiveness, resources and their use, commercial availability and viability, and system integration options. The problem of intense water consumption in ammonia production was also highlighted, which requires further studies to address the associated environmental and economic impacts.

This research topic also addresses the utilization of hydrogen as an energy vector in transportation and power sectors. Ji et al. carried out a design and performance quantification of a compact air-breathing jet hybrid-electric engine coupled with solid oxide fuel cells (SOFCs). Compared with the traditional turbojet engine, this hybrid-electric engine increases the specific thrust by $20.3 \%$ and the thermal efficiency by $6.3 \%$. Schulthoff et al. investigated the role of hydrogen in decarbonizing the power sector. While it is found that the current cost projection of power-to-hydrogen is not competitive to alternative solutions such as pumped hydro and batteries, the authors suggested an international hydrogen-trading system to decrease the hydrogen cost and the potential of using hydrogen in the hard-to-abate sectors and multisectoral applications to maximize its benefits.

In summary, this research topic showcased five different areas of interest to the hydrogen community including sustainable hydrogen production, ammonia as a hydrogen carrier, and hydrogen utilization in transportation and power sectors. As hydrogen is gaining momentum as an energy vector to decarbonize the economy, we expect a significant number of research and demonstrations studies to be reported in the coming decade.

\section{AUTHOR CONTRIBUTIONS}

X-YW drafted the editorial. YL, FH and WL provided constructive feedback and revised the editorial.

\section{FUNDING}

X-YW acknowledges the Start-up Grant of University of Waterloo. YL acknowledges the National Key R\&D Program 
of China for Renewable Energy and Hydrogen Technology (2020YFB1505604) and the National Science Foundation of China, NSFC (No. 22008034/U2005215). WL acknowledges the

\section{REFERENCES}

Council of Australian Governments Energy Council (2019). Australia's National Hydrogen Strategy. Available at: https://www.industry.gov. $\mathrm{au} /$ sites/default/files/2019-11/australias-national-hydrogen-strategy. pdf.

European Commission (2020). A Hydrogen Strategy for a Climate-Neutral Europe. Available at: https://knowledge4policy.ec.europa.eu/ publication/communication-com2020301-hydrogen-strategy-climate-neutraleurope_en.

International Energy Agency (2019). The Future of Hydrogen. Available at: https://www.iea.org/reports/the-future-of-hydrogen. financial support by the Australian Research Council (ARC Future Fellowship FT140101213). FH acknowledges funding by the Fond der Chemischen Industrie through a Liebig Fellowship.

Natural Resources Canada (2020). Hydrogen Strategy for Canada: Seizing the Opportunities for Hydrogen. Available at: https://www.nrcan.gc.ca/climatechange/the-hydrogen-strategy/23080.

Conflict of Interest: The authors declare that the research was conducted in the absence of any commercial or financial relationships that could be construed as a potential conflict of interest.

Copyright (C) $2021 \mathrm{Wu}$, Luo, Hess and Lipinski. This is an open-access article distributed under the terms of the Creative Commons Attribution License (CC BY). The use, distribution or reproduction in other forums is permitted, provided the original author(s) and the copyright owner(s) are credited and that the original publication in this journal is cited, in accordance with accepted academic practice. No use, distribution or reproduction is permitted which does not comply with these terms. 